\title{
Application of a prechop technique using a reverse chopper in small pupil cataract surgery
}

\author{
Ke Yang ${ }^{1}$, Chenjie Song ${ }^{1}$, Jiaxin $\mathrm{Li}^{2} \wedge$, Weihua Zhang ${ }^{3} \wedge$, Zhanjiang Liu $^{4} \wedge$, Yang Zhao ${ }^{1}$ \\ ${ }^{1}$ Beijing Tongren Eye Center, Beijing Key Laboratory of Ophthalmology and Visual Science, Beijing Tongren Hospital, Capital Medical University, \\ Beijing, China; ${ }^{2}$ Medical School, Pingdingshan University, Pingdingshan, China; ${ }^{3}$ Liaocheng People's Hospital, Liaocheng, China; ${ }^{4}$ Chaoyang \\ Central Hospital, Chaoyang, China \\ Contributions: (I) Conception and design: Y Zhao; (II) Administrative support: K Yang; (III) Provision of study materials or patients: C Song, J Li; \\ (IV) Collection and assembly of data: W Zhang, Z Liu; (V) Data analysis and interpretation: J Li; (VI) Manuscript writing: All authors; (VII) Final \\ approval of manuscript: All authors. \\ Correspondence to: Yang Zhao, MD. Dongjiaominxiang 1st, Dongcheng District, Beijing, China. Email: doctor.zy@qq.com.
}

Background: To analyze the value of a prechop technique for splitting the nucleus of the lens using a reverse chopper in small-pupil cataract surgeries. A prospective case-control study. Thirty-four cataract patients (34 eyes) who were treated in our center from March 2019 to December 2019 were enrolled and then divided into two groups: small pupil group (18 patients; 18 eyes) and normal pupil group (16 patients; 16 eyes).

Methods: The prechop technique was applied in both groups, and the patients were followed up for three months. The best-corrected visual acuity (BVCA), surgical complications, corneal endothelial cell loss, pupil function, operative time, and cumulative dissipated energy (CDE) were compared between these two groups, and the safety of the nucleus-chopping technique was evaluated. Data were analyzed using the SPSS 23.0 software packages. BVCA, surgical complications, rate of corneal endothelial cell loss, pupil function, operative time, and CDE.

Results: The surgery was smooth in all cases. The operative time, intraoperative ultrasound energy consumption, BVCA, and surgical complications indicated no significant difference between the two groups (all $\mathrm{P}>0.05$ ). In the small pupil group, BVCA was significantly improved after surgery and achieved its optimal value three months after surgery $\left(\chi^{2}=49.38 ; \mathrm{P}=0\right)$. The diameter of the pupil was about $3.22 \mathrm{~mm}$ in the small pupil group before nucleus chopping. The postoperative pupil morphology was not statistically different from that before surgery (pupil morphology: $\chi^{2}=0.131 ; \mathrm{P}=0.717$ ); however, the pupillary light reflex was significantly improved after surgery $\left(\chi^{2}=8.378 ; \mathrm{P}=0.004\right)$, and the pupil diameter was significantly increased ( $\mathrm{T}=-3.494 ; \mathrm{P}=0.003$ ). The rate of corneal endothelial cell loss was higher in small pupil group than in the normal pupil group in the 3 rd postoperative month, but the difference was not statistically significant $(\mathrm{T}=-0.023 ; \mathrm{P}=0.982)$.

Conclusions: The prechop technique using a reverse chopper in small-pupil cataract surgery occupies a similar operative time, cumulative energy consumption during operation, BVCA, pupil morphology, and rate of corneal endothelial cell loss, comparing with those in normal-pupil cataract surgery. To such a degree, it is a safe, high-efficiency, a simple and easy-to-operate nucleus-chopping technique that can be used in smallpupil cataract surgery.

Keywords: Small-pupil cataract; nucleus splitting technique; reverse chopper; phacoemulsification

Submitted May 01, 2020. Accepted for publication Sep 08, 2020.

doi: $10.21037 /$ atm-20-5241

View this article at: http://dx.doi.org/10.21037/atm-20-5241

\footnotetext{
^ ORCID: Ke Yang: 0000-0002-8354-3136; Chenjie Song: 0000-0003-0997-922X; Jiaxin Li: 0000-0002-1543-3576; Weihua Zhang: 0000-00026988-2318; Zhanjiang Liu: 0000-0002-9727-0394.
} 


\section{Introduction}

Phacoemulsification is the mainstay for cataract treatment, and a wide variety of methods are available to clinicians. The primary treatment for cataracts is phacoemulsification (1), during which the fragmentation and aspiration of the nucleus fractures are the keys to a successful recovery. Usually, dilated pupils are undoubtedly a prerequisite for successful nucleus emulsification. However, phacoemulsification in small pupils can be particularly challenging for most surgeons, and it is also associated with a variety of surgical complications (2). Research has shown that a small pupil is a risk factor for vitreous prolapse in cataract surgery (3). Many small-pupil techniques for phacoemulsification have been developed to address the issue of nucleus chopping in small-pupil cataract surgery; however, most of these techniques require tight cooperation between the hands and feet, and the continuous release of substantial amounts of ultrasound energy during surgery may damage intraocular tissues. Plus, various pupil dilators have developed to lower the surgical difficulty by dilating the pupil to more than $6 \mathrm{~mm}$ before phacoemulsification; however, these instruments require the creation of additional incisions, which makes the procedures even more complicated and increases the expenditure.

The prechop technique significantly reduces the intraoperative use of ultrasound energy, thus reducing loss of corneal endothelium and injuries to other intraocular structures. The prechop technique has been proved useful not only in normal pupil but also in small pupil cataract surgery.

We have developed a novel prechop technique using a reverse chopper (4) before phacoemulsification of the lens nucleus, which achieves efficiently in situ nucleus-splitting without any ultrasonic energy and therefore has advantages including less time and energy during phacoemulsification and fewer surgical complications. We have applied this technique in surgeries for complex cataracts such as hardnucleus cataract and ultra-high myopia $(5,6)$. Compared with conventional nucleus-chopping techniques, nucleussplitting using a reverse chopper retains many advantages in terms of ultrasound time (UST), postoperative visual recovery, and postoperative corneal endothelial cell damage. We also found this technique could be used in cataract surgery with small pupil. Here we report the application of this novel technique in the treatment of small-pupil cataracts.

We present the following article in accordance with the
STROBE reporting checklist (available at http://dx.doi. org/10.21037/atm-20-5241).

\section{Methods}

\section{Subjects}

\section{General data}

Totally 34 cataract patients (34 eyes) who were treated in our center from March 2019 to December 2019 were enrolled in this prospective case-control study. All patients signed informed consent before enrollment. The inclusion criteria are as follows: (I) meeting the indications for cataract surgery; (II) with lens opacity grade 4 [based on the Emery-Little Classification (7)] and intraocular pressure (IOP) of $\leq 21 \mathrm{mmHg}$; (III) the inflammation was in the quiet period (i.e., the inflammation remained stable for at least 6 months); and (IV) with good adherence to treatment and follow-up visits. The exclusion criteria were as follows: (I) with lens subluxation or luxation; (II) with corneal lesions including keratoconus, corneal endothelium $<1,000 /$ $\mathrm{mm}^{2}$, and/or corneal leukoplakia; (III) with a history of macular degeneration, retinal detachment, and/or ocular trauma; and (IV) aged $\leq 18$ years. The ethics committee had approved the study of our center (Approval No.: TRECKY2017-028). All procedures performed in this study involving human participants were in accordance with the Declaration of Helsinki (as revised in 2013). All patients signed the informed consents before enrollment.

\section{Grouping}

Pupils were dilated with topical compound tropicamide eye drops six times one hour before surgery. Patients were defined as in the small pupil group if the light reflex disappeared, and the pupil diameter was $\leq 4 \mathrm{~mm}$ under the slit lamp and as in the normal pupil group if the pupil diameter was $>6 \mathrm{~mm}$. There were 18 patients (18 eyes; 5 males and 13 females aged [71.67 $\pm 10.19 .19]$ years) in the small pupil group, and the causes of undilated pupils included diabetes in 3 cases, synechia after glaucoma surgery in 12 cases, iridocyclitis after glaucoma surgery in 2 cases, and other reason in 1 case. The best-corrected visual acuity (BCVA) ranged $0.01-0.5$ before surgery [values based on the Snellen E chart, which are converted to the logarithm of minimal angle of resolution ( $\log M A R)$ values according to the literature (8) for statistical analysis]. There were 16 patients $[16$ eyes; 4 males and 12 females aged $(73.31 \pm 8.72 .72)$ years] in the normal pupil group, and their 
Table 1 Comparisons of preoperative baseline characteristics between two groups

\begin{tabular}{lccccc}
\hline \multirow{2}{*}{ Group } & $\mathrm{n}$ & Age (years) & \multicolumn{2}{c}{ Gender } & Preoperative logMAR value \\
\cline { 4 - 5 } & & & Males & Female & \\
\hline Small pupil group & 18 & $71.67 \pm 10.19$ & 5 & 13 & $0.61(0.37,1.02)$ \\
Normal pupil group & 16 & $73.31 \pm 8.72$ & 4 & 12 & $0.30(0.30,1.00)$ \\
Statistics & & -0.503 & 0 & -1.850 \\
P value & & 0.619 & 1.000 & 0.064
\end{tabular}

Age was compared by independent sample $t$-test, gender by chi-square test, and preoperative logMAR value by non-parametric test. logMAR, logarithm of minimal angle of resolution.

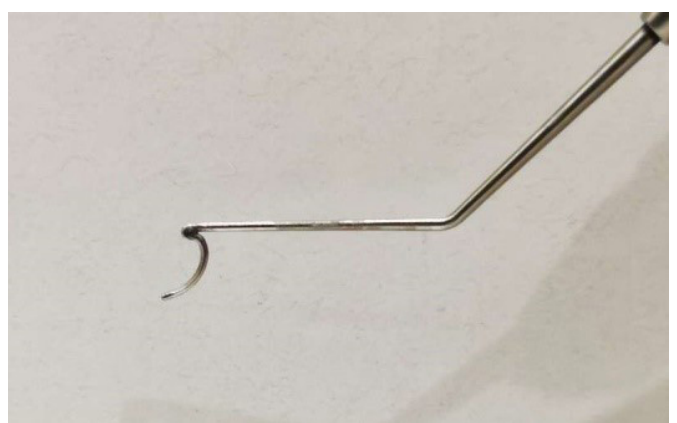

Figure 1 Structure diagram of a reverse chopper.

preoperative BVCA ranged 0.05-0.6.

The age, gender, and BCVA were matched between these two groups before surgery (both $\mathrm{P}>0.05$ ) (Table 1).

\section{Surgical maneuvers}

The same experienced surgeon operated. As described in the literature (4), a reverse mental chopper was used in the phacoemulsification and intraocular lens (IOL) implantation (Figure 1). The reverse chopper was reverse round-arc in shape, with about $2.5-3.0 \mathrm{~mm}$ between two arc tips. The tips were blunt, and there was an inner blade on the arc.

\section{Small pupil group}

(I) Separation of posterior synechia (i) the localized dotted posterior synechia was separated by a viscoelastic agent or bluntly separated by the chopper; (ii) for patients with wide posterior synechia, atrophy of iris stroma, and organized membranes. The iris sphincter was cut open at multiple sites (4-6 sites; the pupil sphincter was divided by $<1 \mathrm{~mm}$ ) to dilate the pupil to a diameter of about $4 \mathrm{~mm}$.
(II) Phacoemulsification combined with IOL implantation (Figure 1): a transparent corneal incision was created at the 10:00 location. The viscoelastic agent was injected into the anterior chamber, and a puncture was made at the 2:00 location. The posterior synechia was separated by employing the earlier method, and curvilinear capsulorhexis was performed. The Nagahara chopper entered the anterior chamber via the lateral incision at the 2:00 location and was fixed in the center of the lens nucleus. The reverse chopper horizontally entered the anterior chamber via the corneal incision at the 10:00 location and was pressed downwards around the nucleus at the opening of the capsular bag. After the chopper was located in the cortex between the lens nucleus and the capsular bag (Figure 2A), the reverse chopper was erected to make the arcuate blade perpendicular to the equator of the lens. With the help of the operator's left hand, the Nagahara chopper slid into the cortex or capsule opposite to the diameter line of the reverse chopper, with its blade being perpendicular to the equator of the lens (Figure 2B). Both hands exerted forces along the radial direction of the lens center. Both choppers horizontally moved towards each other and split the lens nucleus, and then they moved laterally to completely divide the lens nucleus into two semi-ellipsoids (Figure 2C). The later steps included phacoemulsification of lens nucleus (phacoemulsification instrument: ALCON vertical, INFINITI $^{\circledR}$ VISION SYSTEM, USA), aspiration of the cortex, and IOL implantation in the capsular bag. Implantation of the capsular tension rings may also be needed in some cases. After the surgery, the Tobramycin dexamethasone eye ointment was applied to the conjunctival sac, and the eyes were covered with 

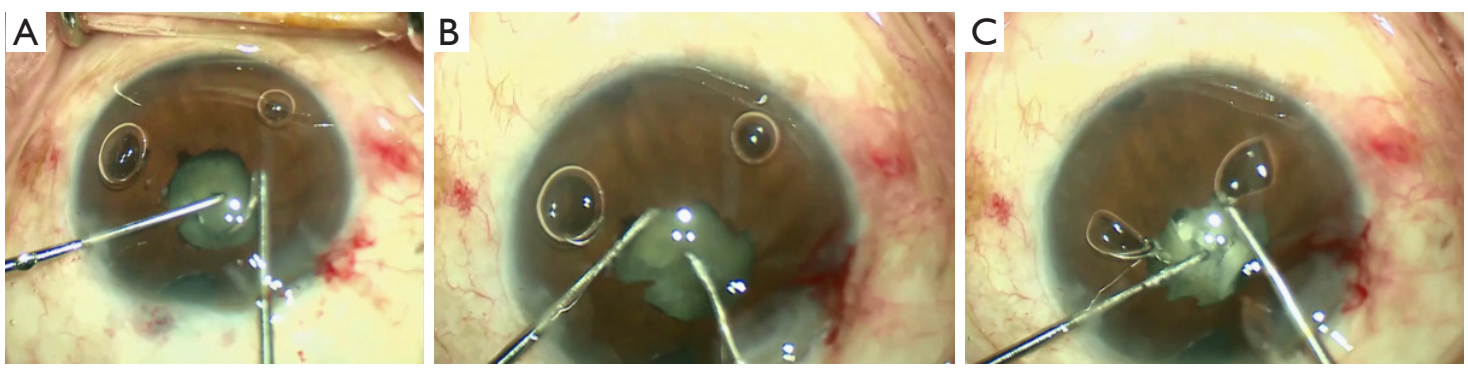

Figure 2 Phacoemulsification combined with IOL implantation. (A) The reverse chopper horizontally entered the anterior chamber and was pressed downwards around the nucleus at the opening of the capsular bag. After the chopper was located in the cortex between the lens nucleus and the capsular bag, the reverse chopper was erected to make the inner arcuate blade perpendicular to the equator of the lens; (B) with the help of the operator's left hand, the Nagahara chopper was moved into the capsular bag and located at the opposing side of the diameter line of the reverse chopper. These two choppers moved towards each other and divided the lens nucleus horizontally; (C) the Nagahara chopper and the reverse chopper laterally separated the nucleus into two semi-ellipses at the center of the lens. IOL, intraocular lens.

eye pads.

The complete surgery video: "Small pupil cataract surgery using a reverse chopper" is attached.

\section{Normal pupil group}

No separation of posterior synechia was needed, and the remaining surgical steps were the same as in the small pupil group.

\section{Main measures and evaluation standards}

The eye's anterior segments, including the hardness of the lens nucleus and the morphology of the pupils, were examined using a slit lamp. The IOP was measured using a non-contact tonometer (TX-20, Canon, Japan). The pupil diameter was calculated using the image measurement software Image $J$ based on the ratio between pupil diameter and corneal diameter (corneal diameter measurement: with the length of a $3.0-\mathrm{mm}$ puncture knife as a reference during the operation), which was obtained by external ocular photography. Fundus photography, ultrasound measurement of the axial length of the eye, measurement of corneal endothelial cells using a cytometer (SP-3000P, TOPCON), and subjective refraction were equally performed. Operative time, UST, cumulative dissipated energy (CDE), and intraoperative complications were recorded. The pupil diameter before nucleus-chopping was measured during the operation. The length of a $3.0-\mathrm{mm}$ puncture knife was used as a reference in the small pupil group. Plus, the BCVA, IOP, pupil size and shape, and complications (e.g., anterior chamber reaction, corneal edema, iris bleeding, posterior capsule rupture, and crystalline nucleus detachment into the vitreous cavity) were recorded 7 days, 1 month, and 3 months after surgery $(9,10)$. The count of corneal endothelial cells was measured 3 months after the operation.

\section{Statistical analyses}

Statistical analysis was performed by using the SPSS 23.0 software package. Shapiro-Wilk test was performed firstly to show the normality of data. Typically (or roughly normally) distributed measurement data are presented as mean \pm standard deviation and analyzed by using independent sample $t$-test and paired $t$-test. Non-normally distributed measurement data are described using median (upper quartile, lower quartile) and analyzed using non-parametric analysis of variance. The count data are presented with frequency (percentage) and analyzed using the chi-square test (including the continuity correction and Fisher's exact probability method). A value of $\mathrm{P}<0.05$ was considered statistically significant, and the test was two-sided.

\section{Results}

\section{Surgeries}

The same surgeon completed all the operations. IOL was implanted into the capsular bag in all cases. No intraoperative complication was noted. 
Table 2 Comparisons of operative time, UST, and CDE between two groups

\begin{tabular}{lllll}
\hline Group & $\mathrm{n}$ & Operative time (seconds) & CDE & UST (seconds) \\
\hline Small pupil group & 18 & $255.00(215.25,351.25)$ & $7.34(3.16,12.03)$ & $29.90(21.43,33.10)$ \\
Normal pupil group & 16 & $246.00(230.00,259.50)$ & $6.55(5.08,10.51)$ & $32.50(26.80,42.48)$ \\
Statistics & & -0.984 & -0.138 & -1.346 \\
P value & & 0.325 & 0.890 & 0.178 \\
\hline
\end{tabular}

Non-parametric test. UST, ultrasound time; CDE, cumulative dissipated energy.

Table 3 Comparison of BCVA after surgery

\begin{tabular}{|c|c|c|c|c|}
\hline Group & $\mathrm{n}$ & $\begin{array}{l}\text { logMAR value one week before } \\
\text { surgery }\end{array}$ & logMAR value 1 month after surgery & logMAR value 3 months after surgery \\
\hline Small pupil group & 18 & $0.26(0.15,0.52)$ & $0.10(0.05,0.33)$ & $0.05(0.00,0.27)$ \\
\hline Normal pupil group & 16 & $0.15(0.10,0.43)$ & $0.00(0.00,0.42)$ & $0.00(0.00,0.56)$ \\
\hline Statistics & & -1.497 & -1.577 & -0.707 \\
\hline
\end{tabular}

Non-parametric test. BCVA, best-corrected visual acuity; logMAR, logarithm of minimal angle of resolution.

\section{Comparisons of operative time, UST, and CDE between two groups}

The operative time, CDE, and UST were shorter or fewer in the normal pupil group than in the small pupil group, but the differences were not statistically significant (operative time: $\mathrm{P}=0.325$; $\mathrm{CDE}: \mathrm{P}=0.890$; UST: $\mathrm{P}=0.178$ ) (Table 2).

\section{Comparison of BCVA and IOP between two groups after the operation}

BCVA showed no significant difference between these two groups after the operation (Table 3). In the small pupil group, BVCA was significantly improved after surgery and achieved its optimal value three months after surgery $\left(\chi^{2}=49.380 ; \mathrm{P}=0\right)$. The IOP was within the normal range after the operation in both groups, and there was no statistical difference between these two groups $(\mathrm{t}=-0.928$, $\mathrm{P}=0.361$ ).

\section{Comparison of corneal edema and corneal endothelial cell loss between two groups after the operation}

The corneal endothelial cell count was not significantly different between the two groups before surgery $(t=-0.302$, $\mathrm{P}=0.765$ ). After the surgery, corneal endothelial cell count decreased in both groups, especially in the small pupil group; however, the rate of corneal endothelial cell loss showed no significant difference between the two groups in the $3 \mathrm{rd}$ postoperative month $(\mathrm{t}=-0.023, \mathrm{P}=0.982)$ (Table 4). Postoperative corneal edema was relatively more severe in small pupil group than in the normal pupil group one week after operation $(\mathrm{P}=0.580)$; it disappeared in both groups three months later $(\mathrm{P}=1)$.

\section{Comparison of postoperative anterior chamber reaction between two groups}

Patients in the small pupil group recovered more slowly than in the normal pupil group. No anterior chamber reaction was observed in the $3 \mathrm{rd}$ postoperative month. However, there was no statistical difference between these two groups ( 7 days after surgery: $\mathrm{P}=0.521 ; 1$ month after surgery: $\mathrm{P}=1)($ Table 5$)$.

\section{Comparison of postoperative pupil status between the two groups}

The pupil diameters showed a significant difference between two groups after mydriasis before surgery $(\mathrm{t}=-13.867, \mathrm{P}=0)$; however, the diameter $(\mathrm{P}=0.698)$; and shape $(\mathrm{P}=0.072)$ of the pupils, as well as the pupillary light reflex $(\mathrm{P}=1)$, showed no significant differences after surgery 
Table 4 Comparison of corneal endothelial cell count between two groups before and after surgery

\begin{tabular}{lcccc}
\hline \multirow{2}{*}{ Group } & $\mathrm{n}$ & \multicolumn{2}{c}{ Corneal endothelial cell count $\left(/ \mathrm{mm}^{2}\right)$} & $\begin{array}{c}\text { Rate of corneal endothelial } \\
\text { cell loss }\end{array}$ \\
\cline { 3 - 4 } & & Before surgery & 3 months after surgery & $24.21 \pm 7.24$ \\
Small pupil group & 18 & $2,477.68 \pm 438.06$ & $1,887.22 \pm 423.08$ & $24.28 \pm 9.43$ \\
Normal pupil group & 16 & $2,528.28 \pm 539.89$ & $1,928.04 \pm 540.23$ & -0.023 \\
Statistics & & -0.302 & -0.247 & 0.982 \\
P value & & 0.765 & 0.807 & 0.98 \\
\hline
\end{tabular}

The pre- and postoperative corneal endothelial cell count was analyzed using an independent sample $t$-test, and the non-parametric test analyzed the rate of corneal endothelial cell loss after surgery).

Table 5 Comparison of postoperative anterior chamber reaction between two groups

\begin{tabular}{|c|c|c|c|c|c|c|}
\hline \multirow[t]{2}{*}{ Group } & \multirow[t]{2}{*}{$\mathrm{n}$} & \multicolumn{3}{|c|}{$\begin{array}{l}\text { Anterior chamber reaction grade } 1 \text { week after } \\
\text { surgery }\end{array}$} & \multicolumn{2}{|c|}{$\begin{array}{c}\text { Anterior chamber reaction grade } 1 \text { month after } \\
\text { surgery }\end{array}$} \\
\hline & & 0 & 1 & 2 & 0 & 1 \\
\hline Normal pupil group & 16 & 14 & 2 & 0 & 16 & 0 \\
\hline$P$ value & & \multicolumn{3}{|c|}{0.521} & \multicolumn{2}{|c|}{1} \\
\hline
\end{tabular}

Chi-square test.

(Table 6). The pupil diameter of the small pupil group was about $3.22 \mathrm{~mm}$ before nucleus chopping. Observations of the pupil shape and the pupillary light reflex in the small pupil group one week after surgery showed that the pupil edges were irregular in 5 eyes. These results were not statistically different from those before surgery (pupil shape: $\mathrm{P}=0.717$ ). Also, the pupillary light reflex was slow in 1 eye, but it was significantly improved after surgery $(\mathrm{P}=0.004)$. Finally, the pupil diameter was significantly increased after surgery $(\mathrm{P}=0.003)$ (Table 7$)$.

\section{Discussion}

A prerequisite for safe and smooth cataract surgery is a dilated pupil. However, the pupil diameter may still be $\leq 4 \mathrm{~mm}$, despite the use of mydriatic in some patients, due to reasons such as glaucoma surgery, recurrent uveitis, long-term use of miotic agents, diabetes, and/or senile dilator pupillae injury) $(11,12)$. The operators often cannot accurately judge the conditions (e.g., the turbidity and hardness of the nucleus) behind the iris when the pupil diameter is small. Under such a condition, lack of predictability and low safety make the cataract surgery a risky and challenging operation (13). The improper nucleus splitting in patients with small pupil increases the intraoperative risks of iris sphincter tear, iris bleeding, posterior capsular tears, nucleus detachment into the vitreous cavity, and decompensation of the corneal endothelium after surgery. Further, it also severely affects postoperative recovery and postoperative pupil morphology and function, causing postoperative photophobia, glare, multiple vision, and other discomforts, and ultimately affect the patient's vision-related quality of life $(2,3,14)$. In recent years, a variety of nucleus chopping techniques (e.g., twohanded nucleus chopping, the high negative pressure in situ nucleus chopping, and deep-buried nuclear splitting) based on the combinations of ultrasound energy with mechanical force have been developed. Although these new techniques have, to a certain extent, improved the nucleus chopping efficiency, they still have the following problems: (I) pupil dilation $>6 \mathrm{~mm}$ is required before the operation; (II) the use of ultrasonic energy before and during the chopping increases both the total ultrasound energy consumption and the damage to intraocular tissues; (III) the chopping 
Table 6 Pupil morphologies in two groups before and after surgery

\begin{tabular}{|c|c|c|c|c|c|c|}
\hline Group & \multicolumn{2}{|c|}{ Pupil diameter (mm) } & \multicolumn{2}{|c|}{ Postoperative pupil shape } & \multicolumn{2}{|c|}{ Pupillary light reflex } \\
\hline Small pupil group & $3.74 \pm 0.59$ & $3.46 \pm 0.71$ & 13 & 5 & 17 & 1 \\
\hline Normal pupil group & $6.58 \pm 0.59$ & $3.55 \pm 0.69$ & 16 & 0 & 16 & 0 \\
\hline Statistics & -13.867 & -0.392 & \multicolumn{2}{|c|}{3.231} & \multicolumn{2}{|c|}{0} \\
\hline
\end{tabular}

Independent sample $t$-test is used for pupil diameter and chi-square test for pupil morphology and pupillary light reflex.

Table 7 Pupil morphologies in small pupil groups before and after surgery

\begin{tabular}{|c|c|c|c|c|c|}
\hline Period & Pupil diameter (mm) & \multicolumn{2}{|c|}{ Pupil shape } & \multicolumn{2}{|c|}{ Pupillary light reflex } \\
\hline Before surgery & $2.99 \pm 0.71$ & 12 & 6 & 8 & 10 \\
\hline After surgery & $3.46 \pm 0.71$ & 13 & 5 & 1 & 17 \\
\hline Statistics & -3.494 & \multicolumn{2}{|c|}{0.131} & \multicolumn{2}{|c|}{8.378} \\
\hline
\end{tabular}

Independent sample $t$-test is used for pupil diameter and chi-square test for pupil morphology and pupillary light reflex.

force in the centrifugal direction increases the pressure on the ligament, and the use of such techniques in a limited space depends highly on the operator's two-handed skills and on his/her foot pedal control, which hampers the clinical promotion of these techniques (15). Some authors have proposed that the pupil can be temporarily dilated to more than $6 \mathrm{~mm}$ using a pupil dilator to complete the surgery; however, problems persist. For instance, the use of a disposable pupil dilator and its special auxiliary implants increase the cost of surgery; also, the implantation, placement, and removal of the pupil dilator can easily damage the corneal endothelial cells. Therefore, we need a safer, more efficient, and simpler nucleus chopping technique. How to properly deal with pupils and how to efficiently and safely complete a small pupil cataract surgery in a limited operating space by using an appropriate nucleus chopping technique has become hot research topics.

The pre-chop technique significantly reduces the intraoperative use of ultrasound energy, thus reducing loss of corneal endothelium and injuries to other intraocular structures. The main manual pre-chop techniques currently published have limitations in small pupil cataract surgery that restrict the wide application of the pre-chop technique.

The prechop technique using a reverse chopper uses the reverse chopper and the Nagahara chopper to horizontally divide the lens nucleus into $2-3$ parts in the capsular bag by using a centripetal force before completing the phacoemulsification without using any ultrasonic energy. After the nucleus is chopped, the nuclei become smaller and can be aspirated out from the smaller pupil, which enables a safe and smooth surgery when the pupil diameter is about $4 \mathrm{~mm}$. In our current study, we compared the application of this technique in cataract patients with either small pupils or normal pupils. It was found that the small pupil group had significantly higher postoperative corneal endothelial cell loss rate and slower recovery of corneal edema, which might be explained by the shallower anterior chamber depth and smaller operating space in patients with small pupils. Although efforts had been made to perform phacoemulsification and IOL implantation far away as possible from the corneal endothelium, the impacts of these steps on the corneal endothelium were still more evident than those in the normal pupil group. However, there was no significant difference between these two groups in ultrasound energy use as well as BCVA and corneal endothelial cell loss rate 3 months after surgery. Also, we found that pupillary light reflex recovered within 1 week after surgery in $94.44 \%$ of patients with small pupils. However, pupil diameter increased after surgery in most patients, and $28 \%$ of the patients had 1 to 6 small zigzag 
defects at their pupil margins, with the pupils being nearly round. Most patients in our small pupil group underwent blunt dissection to remove posterior synechia, which was conducive to the restoration of the pupil function. Although the chopper can exert a pulling force on the edge of the pupil during chopper placement and nucleus chopping, such force is feeble and, therefore, will not affect pupil function. In a small proportion of patients, however, the posterior synechia can be extensive and diffuse before surgery, and some organized membranes may even present in the pupil area. The pupil diameter is only $2-3 \mathrm{~mm}$ even after mydriasis and/or blunt separation with viscoelastic agents. For such tiny pupils, multi-site radial dissection of the pupillary sphincter was performed by us to radially cut open the pupil sphincter (with a width of $<1 \mathrm{~mm}$ ) at 4-6 sites (the pupil diameter after pupil dilation was about 3-4 mm), which did not damage all pupil sphincter muscles. Although the residual incision on the margin of the pupil after the operation resulted in the pupil being not round, the overall function of the pupil sphincter was not affected, which can effectively retain the physiological function of the pupil. In our current series, the postoperative pupil function demonstrated no significant difference between the two groups.

In summary, this novel technique possesses the following advantages: (I) design of the chopper: the nondisposable design allows the reuse of the chopper after sterilization, which lowers the surgical expenditure. The tip of the nucleus chopper is round and blunt, and thus the capsular bag can be protected during nucleus chopping and displacement. The capsule will not be injured even when pushing the equator. The inner blade on the inner side of the chopper holds the nucleus firmly, and the chopping efficiency is high. (II) The chopping process: the choppers enter the lens via the main incision, and the nucleus is squeezed forcefully in the horizontal direction. It prevents any change in the suspension ligament and reduces the risk of suspension ligament rupture. (III) Applicable conditions: This technique allows the safe placement of the choppers under non-direct vision, even if there is no direct view of the large pupils. The visible range is not a determining factor. The operator can receive adequate training on normal pupils and then applies this technique in small-pupil surgery, and the learning curve is short $(14,16)$.

Although this technique is safe and effective, caution is however required during its clinical application. First, the choppers should be placed in both hands and wellfixed in the capsular bag of the lens' equatorial region before forceful chopping towards each side during the prechop. Second, all the surgeries in our current study were completed in pupils sized about $4-5 \mathrm{~mm}$. Due to the small sizes of these pupils and the limited visual range, radial tear of the anterior capsule, posterior capsule rupture, and residual lens cortex may occur after improper maneuvers during capsulorhexis and injection and suction of fluids in the cortex. Thus, the operator must be experienced and skillful.

The purpose of our current study was to analyze the effectiveness of this technique in small-pupil cataract surgery. However, we did not compare it with the currently applicable chopping techniques in small-pupil cataract surgeries in a controlled design; plus, the limited sample size might lead to biases in statistical analysis. With the optimization of this technique, more surgeons will acquire the relevant surgical skills by performing the surgery in more cases; as a result, the advantages and disadvantages of this technique will be identified in studies with more cases. A well-designed randomized controlled study is warranted to verify the clinical effectiveness of this technique further.

\section{Acknowledgments}

Funding: None.

\section{Footnote}

Reporting Checklist: The authors have completed the STROBE reporting checklist. Available at http://dx.doi. org/10.21037/atm-20-5241

Data Sharing Statement: Available at http://dx.doi. org/10.21037/atm-20-5241

Conflicts of Interest: All authors have completed the ICMJE uniform disclosure form (available at http://dx.doi. org/10.21037/atm-20-5241). The authors have no conflicts of interest to declare.

Ethical Statement: The authors are accountable for all aspects of the work in ensuring that questions related to the accuracy or integrity of any part of the work are appropriately investigated and resolved. The ethics committee had approved the study of our center (Approval No.: TRECKY2017-028). All patients signed the informed consents before enrollment. All procedures performed in this study involving human participants were in accordance 
with the Declaration of Helsinki (as revised in 2013).

Open Access Statement: This is an Open Access article distributed in accordance with the Creative Commons Attribution-NonCommercial-NoDerivs 4.0 International License (CC BY-NC-ND 4.0), which permits the noncommercial replication and distribution of the article with the strict proviso that no changes or edits are made and the original work is properly cited (including links to both the formal publication through the relevant DOI and the license). See: https://creativecommons.org/licenses/by-nc-nd/4.0/.

\section{References}

1. Li MQ, Ying AX. Phacoemulsification for complex cataract. Road to Health 2017;(06):70.

2. Canabrava S, Rezende PH, Eliazar GC, et al. Efficacy of the Canabrava Ring (pupil expansion device) in cataract surgery for eyes with small pupils: the first 30 cases. Arq Bras Oftalmol 2018;81:202-11.

3. Zare M, Javadi MA, Einollahi B, et al. Risk Factors for Posterior Capsule Rupture and Vitreous Loss during Phacoemulsification. J Ophthalmic Vis Res 2009;4:208-12.

4. Zhao Y, Li J, Yang K, et al. A Prechop Technique Using a Reverse Chopper. J Invest Surg. 2019;32:199-207.

5. Yang K, Zhu SQ, Zhao Y. A randomized controlled clinical study on reverse-chopper prechop technique with phacoand-chop technique for high myopia associated with hard nucleus cataract. Chinese Journal of Experimental Ophthalmology 2017;35:629-33.

6. Zhao Y, Yang K, Li J, et al. Comparison between the prechopping method with a reverse chopper and the

Cite this article as: Yang K, Song C, Li J, Zhang W, Liu Z, Zhao Y. Application of a prechop technique for splitting the lens nucleus using a reverse chopper in small-pupil cataract surgery. Ann Transl Med 2020;8(18):1189. doi: 10.21037/atm20-5241 routine stop-and-chop method in treating cataract with grade IV hard nucleus. J Fr Ophtalmol 2018;41:315-20.

7. Spadea L, Iozzo N. EBOV-RNA test with RT-PCR in ocular fluids of Ebola survivors can help to safely perform cataract surgery. Ann Transl Med 2018;6:S29.

8. Xie Y, Chen JQ, Xu K, et al. Characteristics of OPA1 genotype in Chinese patients with suspected hereditary optic atrophy. Ophthalmic in China 2015;(2):79-84.

9. Yang PZ, Wang H, Huang XK, et al. Quantitative determination of aqueous flare and cells in the eyes of patients with inflammation of anterior uvea. Zhonghua Yan Ke Za Zhi 2004;40:510-3.

10. Xie LX, Yao Z, Huang YS, et al. Corneal endothelial damage and its repair after phacoemulsification. Zhonghua Yan Ke Za Zhi 2004;40:90-3.

11. Halkiadakis I, Chatziralli I, Drakos E, et al. Causes and management of small pupil in patients with cataract. Oman J Ophthalmol 2017;10:220-4.

12. Yao K. Surgeries in Complicated Cases of Cataract. Beijing: Beijing Science and Technology Press, 2008.

13. Papaconstantinou D, Kalantzis G, Brouzas D, et al. Safety and efficacy of phacoemulsification and intraocular lens implantation through a small pupil using minimal iris manipulation. Clin Interv Aging 2016;11:651-7.

14. Kershner RM. Management of the small pupil for clear corneal cataract surgery. J Cataract Refract Surg 2002;28:1826-31

15. Jacob S. Different methods of chopping in cataract surgery. Curr Opin Ophthalmol 2019;30:25-38.

16. Malyugin B. Cataract surgery in small pupils. Indian J Ophthalmol 2017;65:1323-28. 